\title{
Attenuation of exogenous angiotensin II stress-induced damage and apoptosis in human vascular endothelial cells via microRNA-155 expression
}

\author{
TE LIU, DINGZHU SHEN, SANLI XING, JIULIN CHEN, ZHIHUA YU, JIAN WANG, BEILING WU, \\ HUIYING CHI, HONGBIN ZHAO, ZHENZHEN LIANG and CHUAN CHEN
}

Shanghai Geriatric Institute of Chinese Medicine, Longhua Hospital, Shanghai University

of Traditional Chinese Medicine, Shanghai 200031, P.R. China

Received August 21, 2012; Accepted October 18, 2012

DOI: 10.3892/ijmm.2012.1182

\begin{abstract}
Numerous studies have indicated that cells and tissues have means of blocking their response to continuous stress signals to protect themselves from damage. Overexpression of angiotensin II (Ang II) in the renin-angiotensin system can cause vascular endothelial damage, but the mechanism of adjustment of the dynamic equilibrium remains unclear. In this study, we investigated whether microRNA-155 (miR-155) can suppress continuous Ang II stress signals that would otherwise cause vascular endothelial damage. We isolated and cultured human umbilical vein endothelial cells (HUVECs) and transfected one group of these with a mature miR-155 expression plasmid. Quantitative real-time PCR (qRT-PCR) and western blotting showed Ang II type 1 receptor expression to be decreased in miR-155-transfected HUVECs compared with untransfected cells. The MTT proliferation assay revealed that exogenous Ang II suppressed proliferation of HUVECs in a concentration-dependent manner. When HUVECs were cultured in medium containing Ang II at the half maximal inhibitory concentration $(68.94 \mathrm{ng} / \mu \mathrm{l})$ for $24 \mathrm{~h}$, qRT-PCR and western blotting showed that expression of the apoptosis inhibitor Bcl-2 in the HUVEC-Ang II group was markedly lower than that in controls, but apoptosis-promoting factors (Bax, cytochrome $c$, caspases-9 and -3 ) were not. Co-immunoprecipitation western blotting and immunofluorescence staining showed that exogenous Ang II increased the phosphorylation and activation of extracellular signal related kinase (ERK)1/2. Exogenous Ang II also influenced HUVEC migration and capillary tubule formation in vitro. However, after transfection of HUVECs with miR-155 under the same
\end{abstract}

Correspondence to: Dr Chuan Chen, Shanghai Geriatric Institute of Chinese Medicine, Longhua Hospital, Shanghai University of Traditional Chinese Medicine, 558 Xiangyang Road, Shanghai 200031, P.R. China

E-mail: ch9453@126.com

Key words: microRNA-155, angiotensin, vascular endothelial damage, capillary tube formation, migration conditions, expression of apoptosis-promoting factors and ERK1/2 phosphorylation were reduced significantly and HUVEC migration and capillary tubule formation were restored to some extent. Thus, miR-155 attenuated the effect of exogenous Ang II-induced ERK1/2 activation to reduce HUVEC damage and apoptosis. Moreover, miR-155 maintained HUVEC migration and capillary tubule formation in vitro.

\section{Introduction}

Studies indicate that angiotensin and angiogenic factors in the renin-angiotensin system are important early markers of cardiovascular disease (1-3). Angiotensin II (Ang II) plays critical roles in the pathogenesis of pre-eclampsia, acting on endothelial cells, vascular smooth muscle cells and immune cells to accelerate the development of the condition at least partly through low grade inflammation. Ang II stimulates vascular smooth muscle cells to produce monocyte chemoattractant protein, a major chemokine involved in macrophage infiltration. Macrophages, which accumulate in cardiac and other tissues, produce angiotensin-converting enzyme, renin and Ang II (4-6). Continuous stress induced by Ang II can lead to damage and apoptosis in vascular endothelial cells, eventually causing blood vessel damage $(1,2)$. Thus, preventing continuous Ang II stress is important in protecting blood vessels.

A number of studies have reported that the cardiovascular system is a particularly rich source of microRNAs (miRNAs) with roles in disease, which perhaps reflects the susceptibility of the heart and blood vessels to injury and the dependence of mammals on persistent cardiovascular function $(1-3,7)$. miRNAs are 20-23-nucleotide (nt) non-coding RNAs that function as sequence-specific regulators of gene expression through translational repression and/or transcript cleavage (2,7-11). They have recently been discovered to play a major role in gene regulation, including in mammalian cells. Characterization of miRNAs has gained much attention in biology and medicine, with implications for the detection and treatment of different pathologies. miRNAs are abundant in the vascular system, where they have key roles in development 
and are likely to be important mediators of vascular system diseases (2). Thum et al (13) reported that miR-21 is regulated by mitogen-activated protein kinase (MAPK)/extracellular signal related kinase (ERK) signaling in response to cardiac stress $(1,12,14)$. Gao et al (6) suggested that miR-155 overexpression in vascular cells attenuates Ang II-induced $\alpha$-smooth muscle actin expression, and demonstrated that miR-155 regulated adventitial fibroblast differentiation and contributed to suppression of Ang II type 1 receptor (AtR1) expression. Liu et al (9) also showed that lack of miR-155 expression in the human umbilical vein endothelial cells (HUVECs) of puerperant women with pre-eclampsia can lead to high levels of endogenous AtR1 expression and ERK1/2 activation. In view of this evidence, we believe that miR-155 and Ang II are involved in diseases of the blood vessels.

In this study, we isolated and cultured HUVECs from healthy puerperant women and transfected them with an miR-155 expression vector to determine whether this miRNA can regulate the cellular response to Ang II stress signaling by targeting AtR1.

\section{Materials and methods}

HUVECs. The human umbilical cords of healthy puerperant women were collected in asepsis after parturition. The HUVECs were isolated by treatment with $1 \%$ Trypsin, as previously described (7). The HUVECs were grown on $1 \%$ gelatin-coated culture plates in McCoy's 5A (SigmaAldrich, St. Louis, MO, USA) supplemented with $15 \%$ fetal bovine serum (Hyclone), $100 \mathrm{U} / \mathrm{ml}$ penicillin and $100 \mu \mathrm{g} / \mathrm{ml}$ streptomycin (Hyclone), at $37^{\circ} \mathrm{C}$ in a humidified atmosphere of air containing $5 \% \mathrm{CO}_{2}$. The cells were used to within 2-3 passages and were identified as endothelial by their cobblestone monolayer appearance in culture and by the expression of cell-surface antigens CD34 and CD31, as detected by flow cytometry (FCM) assay.

Vector construction. For expression plasmid pRNAT-CMV32miR155 (pre-miRNA of miR-155 expression element), oligonucleotide pairs for pre-miRNA of miR-155 and linker sequences with BamHI and XhoI sites were chemically synthesized by GenePharma Co., Ltd. (Shanghai, China). The sequences of the oligonucleotides were: top strand, 5'-GT ggatccCTGTTAATGCTAATCGTGATAGGGGTTTTTGC CTCCAACTGACTCCTACATATTAGCATTAACAGctcgag CC-3', and bottom strand, 5'-GGctcgagCTGTTAATGCTAAT ATGTAGGAGTCAGTTGGAGGCAAAAACCCCTATCAC GATTAGCATTAACAGggatccAC-3' (sequences corresponding to miR-155 seed sequences in capitalized, underlined and bold, and restriction enzyme sites in lower case and underlined). To built the expression plasmid the pairs of oligos were annealed and inserted into the multiple cloning sites between of BamHI and XhoI sites in the pRNAT-CMV32/Neo vector (GenScript, Piscataway, NJ, USA). The negative control plasmid pRNAT-CMV32-miR155-Mut were similarly built, except that 11 nucleotides in sequences corresponding to miR-155 see sequences were mutated (change TTAATGCTAAT to TaAtTcCaAtT, mutations shown in lower-case). Co-transfection of HUVECs from patients or healthy women were conducted to transfer $0.3 \mu \mathrm{g}$ miR-155 expressed vector or miR-155 mutant vector, respectively, with Lipofectamine 2000 reagent according to the manufacturer's protocol. The cells were seeded in a 6-well plate in McCoy's 5A (Sigma-Aldrich) supplemented with $15 \%$ fetal bovine serum, $100 \mathrm{U} / \mathrm{ml}$ penicillin and $100 \mu \mathrm{g} / \mathrm{ml}$ streptomycin (were from Hyclone), at $37^{\circ} \mathrm{C}$ in a humidified atmosphere of air containing $5 \% \mathrm{CO}_{2}$, until $80 \%$ confluent.

Luciferase report assay. All steps of this assay were performed as previously described $(6,7)$. NIH-3T3 cells were seeded at $3 \times 10^{4} /$ well in 48 -well plates and co-transfected with $400 \mathrm{ng}$ of pRNAT-CMV32-miR-155 or pRNAT-CMV32 or pRNATCMV32-miR155-Mut, 20 ng of pGL-ATR1-3UTR-WT or pGL-ATR1-3UTR-Mut, and pRL-TK (Promega, Madison, WI, USA) using Lipofectamine 2000 reagent according to the manufacturer's protocol. After $48 \mathrm{~h}$ transfection, luciferase activity was measured using the dual-luciferase reporter assay system (Promega).

MTT assay for cell proliferation. Each group of HUVECs was seeded at $2 \times 10^{3} /$ well in 96-well plates and cultured in McCoy's $5 \mathrm{~A}$ supplemented with $15 \%$ fetal bovine serum at $37^{\circ} \mathrm{C}$ with $5 \% \mathrm{CO}_{2}$, until $85 \%$ confluent. MTT (Sigma-Aldrich) reagent $(5 \mathrm{mg} / \mathrm{ml})$ was added to the maintenance cell medium at different time points, and incubated at $37^{\circ} \mathrm{C}$ for an additional $4 \mathrm{~h}$. The reaction was terminated with $150 \mu \mathrm{l}$ dimethylsulfoxide (DMSO; Sigma-Aldrich) per well and the cells were lysed for $15 \mathrm{~min}$; the plates were gently shaken for $5 \mathrm{~min}$. Absorbance values were determined by using the enzyme linked immunosorbent assay (ELISA) reader (Model 680; Bio-Rad) at $490 \mathrm{~nm}$.

RNA extraction and analysis by quantitative real-time PCR ( $q R T-P C R)$. Total RNA from each cell was isolated using TRIzol reagent (Invitrogen) according to the manufacturer's protocol. The RNA samples were treated with DNase I (SigmaAldrich), quantified, and reverse-transcribed into cDNA using the ReverTra Ace- $\alpha$ First Strand cDNA Synthesis kit (Toyobo). qRT-PCR was conducted using a RealPlex4 real-time PCR detection system from Eppendorf Co., Ltd. (Germany), with SYBR-Green Realtime PCR Master MIX (Toyobo) used as the detection dye. qRT-PCR amplification was performed over 40 cycles with denaturation at $95^{\circ} \mathrm{C}$ for $15 \mathrm{sec}$ and annealing at $58^{\circ} \mathrm{C}$ for $45 \mathrm{sec}$. Target cDNA was quantified using the relative quantification method. A comparative threshold cycle $(\mathrm{Ct})$ was used to determine gene expression relative to a control (calibrator) and steady-state mRNA levels are reported as an $\mathrm{n}$-fold difference relative to the calibrator. For each sample, the marker gene $\mathrm{Ct}$ values were normalized using the formula $\Delta \mathrm{Ct}=\mathrm{Ct}$ _genes-Ct_18sRNA. To determine relative expression levels, the following formula was used $\Delta \Delta \mathrm{Ct}=\Delta \mathrm{Ct}$ _sample group- $\Delta \mathrm{Ct}$ _control_group. The values used to plot relative expressions of markers were calculated using the expression $2^{-\Delta \Delta \mathrm{Ct}}$. The mRNA levels were calibrated based on levels of 18 sec rRNA. The cDNA of each gene was amplified using primers as previously described $(7,15)$.

Western blot analysis. Total proteins extracts of each group cells were resolved by $12 \%$ SDS-PAGE and transferred on PVDF (Millipore) membranes. After blocking, the PVDF membranes were washed four times for $15 \mathrm{~min}$ with TBST 
at room temperature and incubated with primary antibody. Following extensive washing, membranes were incubated with secondary peroxidase-linked goat anti-rabbit IgG (1:1,000; Santa Cruz Biotechnology, Inc., Santa Cruz, CA, USA) for $1 \mathrm{~h}$. After washing four times for $15 \mathrm{~min}$ with TBST at room temperature once more, the immunoreactivity was visualized by enhanced chemiluminescence (ECL kit; Pierce Biotechnology), and membranes were exposed to Kodak XAR-5 film (Sigma-Aldrich).

Northern blot analysis. All steps of northern blotting were carried out as previously described (7-11). For all groups, $20 \mu \mathrm{g}$ of good quality total RNA was analyzed on a $7.5 \mathrm{M}$ ureum $12 \%$ PAA denaturing gel and transferred to a Hybond $\mathrm{N}^{+}$nylon membrane (Amersham, Freiburg, Germany). Membranes were crosslinked using UV light for $30 \mathrm{sec}$ at $1,200 \mathrm{mjoule} / \mathrm{cm}^{2}$. Hybridization was performed with the miR-155 antisense starfire probe, 5'-CCCCTATCACGATTAGCATTAA-3' (Integrated DNA Technologies, Inc., Coralville, IA, USA), to detect the 22-nt miR-155 fragments according to the manufacturer's instructions. After washing, membranes were exposed for 20-40 h to Kodak XAR-5 film (Sigma-Aldrich). As a positive control, all membranes were hybridized with a human U6 snRNA probe, 5'-GCAGGGGCCATGCTAATCTTCTCTG TATCG-3'. Exposure times for the U6 control probe varied between 15 and $30 \mathrm{~min}$.

Flow cytometric (FCM) analysis of cell cycle by PI staining. Each group cells was seeded at $3 \times 10^{5} /$ well in 6-well plates and cultured until $85 \%$ confluent. Each group cells was washed by PBS three times and were then collected by centrifugation (Allegra X-22R; Beckman Coulter) at 1,000 x $\mathrm{g}$ for $5 \mathrm{~min}$. The cell pellets were the resuspended in $1 \mathrm{ml}$ of PBS, fixed in $70 \%$ ice-cold ethanol, and kept in a freezer $>48 \mathrm{~h}$. Prior to FCM analysis, the fixed cells were centrifuged, washed twice with PBS, and resuspended in PI staining solution (Sigma-Aldrich) containing $50 \mu \mathrm{l} / \mathrm{ml} \mathrm{PI}$ and $250 \mu \mathrm{g} / \mathrm{ml}$ RNase A (SigmaAldrich). The cell suspension, which was hidden from light, was incubated for $30 \mathrm{~min}$ at $4^{\circ} \mathrm{C}$ and analyzed using the FACS (BD FACSAria; BD Biosciences). A total of 20,000 events were acquired for analysis using CellQuest software.

Co-immunoprecipitation assay. All group cells were seeded at $3 \times 10^{5} /$ well in 6 -well plates and cultured until $85 \%$ confluent; they were lysed (500 $\mu \mathrm{l} /$ plate) in a modified cell lysis buffer for western blotting and IP (20 mM Tris, pH 7.5, $150 \mathrm{mM} \mathrm{NaCl}, 1 \%$ Triton X-100, $1 \mathrm{mM}$ EDTA, sodium pyrophosphate, $\beta$-glycerophosphate, $\mathrm{Na}_{3} \mathrm{VO}_{4}$ and leupeptin) (Beyotime Institute of Biotechnology). Following lysis, each sample was centrifuged to clear the lysate of the insoluble debris and pre-incubated with $20 \mu \mathrm{g}$ protein A agarose beads (Beyotime Institute of Biotechnology) by rocking for $30 \mathrm{~min}$ at $4^{\circ} \mathrm{C}$, followed by centrifugation and transfer to a fresh $1.5 \mathrm{ml}$ tube. The rabbit anti-human ERK1/2 polyclonal antibody (Cell Signaling Technology, USA) was incubated for 90 min before re-addition of $20 \mu \mathrm{g}$ protein A agarose beads to capture the immune complexes. The pelleted beads were then washed three times with $500 \mu \mathrm{l}$ cell lysis buffer, dissolved in $4 \mathrm{X}$ SDS-PAGE sample loading buffer, and heated for $10 \mathrm{~min}$ at $95^{\circ} \mathrm{C}$.
Immunofluorescence staining analysis of relative protein expression. The cultured cells were washed three times with PBS and fixed with $4 \%$ paraformaldehyde (Sigma-Aldrich) for $30 \mathrm{~min}$. After blocking, the cells were incubated first with rabbit anti-human Angiotensin II polyclonal antibody (1:200;) and rabbit anti-human ERK1/2 or pERK1/2 polyclonal antibody (1:200; were from Santa Cruz Biotechnology, Inc.) overnight at $4^{\circ} \mathrm{C}$, and then with FITC- or Cy3-conjugated goat anti-rabbit IgG antibody (1:200; Abcam, Cambridge, UK) and $5 \mathrm{mg} / \mathrm{ml}$ DAPI (Sigma-Aldrich) at room temperature for $30 \mathrm{~min}$. Then the cells were thoroughly washed with TBST and viewed through a fluorescence microscope (DMI3000; Leica, Allendale, NJ, USA).

Mitochondrial membrane potential $(\Delta \Psi m)$. JC-1 probe (Beyotime Biotechnology, Shanghai, China) was employed to measure mitochondrial membrane potential $(\Delta \Psi m)$ depolarization. All steps of northern blotting were according to previous reports $(15,16)$. Briefly, cells cultured in 6-well plates after indicated treatments were incubated with an equal volume of JC-1 staining solution $(5 \mu \mathrm{g} / \mathrm{ml})$ at $37^{\circ} \mathrm{C}$ for $20 \mathrm{~min}$ and rinsed twice with PBS. $\Delta \Psi_{m}$ was monitored by determining the relative amounts of dual emissions from mitochondrial JC-1 monomers or aggregates using a fluorescence microscope (DMI3000; Leica) under Argon-ion $488 \mathrm{~nm}$ laser excitation. Mitochondrial depolarization is indicated by an increase in the green/red fluorescence intensity ratio.

Capillary tubule formation assay. All steps of northern blotting were performed according to previous reports $(16,17)$. In brief, HUVECs were plated on matrigel-coated 6 chamber slides $\left(2 \times 10^{5}\right.$ cells/chamber $)$ in the presence and absence of various test substances described in the previous section for the cell migration assay. After $6 \mathrm{~h}$ of incubation in a $\mathrm{CO}_{2}$ incubator, cells were photographed. To quantitate the data, the number of branch points in four non-overlapping fields was determined.

Statistical analysis. Each experiment was performed at least three times, and data are shown as the means $\pm \mathrm{SE}$, where applicable, and differences were evaluated using the Student's t-test. $\mathrm{P}<0.05$ was considered to indicate statistically significant differences.

\section{Results}

miR-155 interferes with ATR1 expression. According to the miRBase Target database (http:/www.mirbase.org), the 3' untranslated region (3'-UTR) of AtR1 mRNA harbors 9-16 putative miRNA target sites, depending on the species analyzed. We focused on human miR-155, which may target the human AtR1 3'-UTR, since these sites are conserved to varying degrees across species (Fig. 1). To identify possible miR-155 target genes, the 3'-UTR sites of AtR1 were cloned on a luciferase activity assay plasmid. Plasmid DNA for wild-type and mutant AtR1 mRNA 3'-UTR and a blank plasmid were co-transfected with a miR-155 expression vector (wild-type, mutant and blank) in NIH-3T3 cells to determine whether AtR1 gene expression is regulated by mature miR-155. The luciferase activity of the AtR1 3'-UTR was significantly 

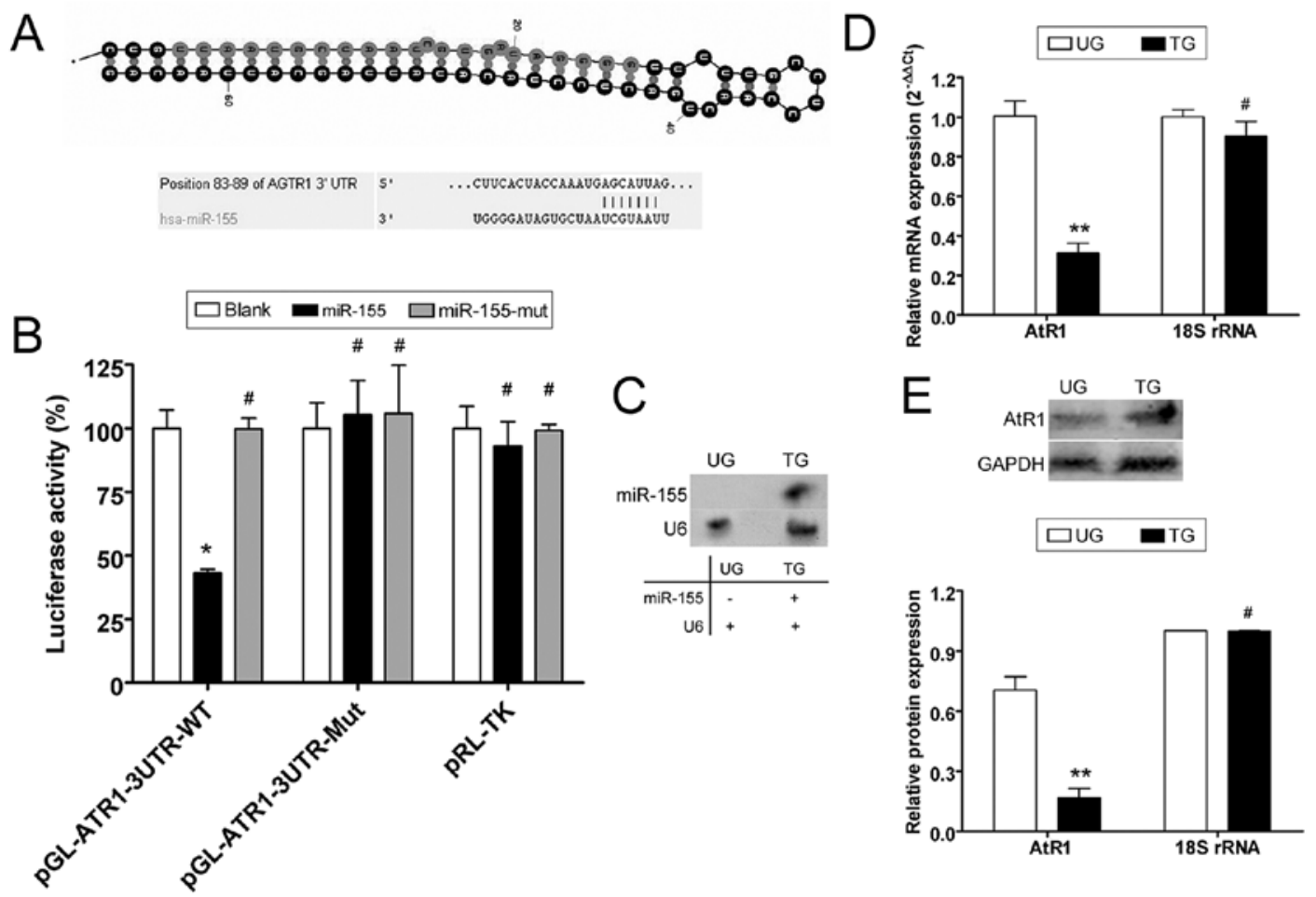

Figure 1. The human AtR1 mRNA 3'-UTR harbors miR-155 binding sites that interfere in the expression of AtR1. (A) The typical secondary structure of precursor miRNAs (pre-miRNAs), illustrated by hsa-miR-155. Stem-loop and hairpin structures are present in the pre-miRNA, and the common binding site is located in an unstable region with a multi-branching, loop-like RNA structure. Gray indicates the mature miRNA that binds to the 3'-UTR of target genes. There is complementarity between miR-155 and a putative human AtR1 3'-UTR target 70-90 bp downstream; conserved bases of the target sequence are shown. (B) A luciferase assay was used to determine miRNA-155 expression and interference with the target AtR1 gene. Wild-type reporter or mutated control luciferase plasmids were transfected into NIH-3T3 cells with miR-155 expression plasmids or miR-155 mutant expression plasmids. Luciferase activity at the AtR1 3'-UTR sites was significantly inhibited by miR-155. ${ }^{*} \mathrm{P}<0.05$ vs. blank; ${ }^{*} \mathrm{P}>0.05$ vs. blank; $\mathrm{n}=3$. (C) Northern blotting showing miR-155 to be expressed in HUVECs transfected with the miR-155 expression plasmid and in untransfected cells. U6 was used as a loading control. The hybridization signal of mature miR-155 was observed in miR-155-transfected HUVECs but not in untransfected cells. (D) qRT-PCR was used to determine whether miR-155 could interfere with AtR1 mRNA expression after transfection into HUVECs. AtR1 mRNA expression was inhibited in HUVECs transfected with the miR-155 expression plasmid compared with untransfected cells. ${ }^{* *} \mathrm{P}<0.01$ vs. UG; ${ }^{*} \mathrm{P}>0.05$ vs. UG; $\mathrm{n}=3$. (E) Western blotting was used to determine whether miR-155 could interfere with AtR1 mRNA expression after transfection into HUVECs. AtR1 protein expression was significantly reduced in miR-155-transfected HUVECs compared with untransfected cells. ${ }^{* *} \mathrm{P}<0.01$ vs. UG; "P>0.05 vs. UG; $\mathrm{n}=3$. UG, untransfected group; TG, HUVECs transfected with miR-155.

Table I. Different cellular growth inhibition and exogenous Ang II concentration detected by MTT assay.

\begin{tabular}{ccc}
\hline Exogenous Ang II concentration $(\mathrm{ng} / \mu \mathrm{l})$ & HUVEC-Ang II group $(\mathrm{n}=3)$ & HUVEC-PBS group $(\mathrm{n}=3)$ \\
\hline 5 & $2.53 \pm 1.11$ & $2.14 \pm 1.47$ \\
10 & $10.36 \pm 1.55$ & $1.96 \pm 2.25$ \\
20 & $10.72 \pm 1.72$ & $2.03 \pm 1.33$ \\
40 & $22.44 \pm 0.21$ & $0.84 \pm 0.76$ \\
80 & $44.17 \pm 2.93$ & $1.71 \pm 0.41$ \\
120 & $51.06 \pm 0.70$ & $2.47 \pm 2.47$ \\
\hline
\end{tabular}

Ang II, angiotensin II; HUVEC, human umbilical vein endothelial cell.

inhibited by miR-155 (Fig. 1), whereas that of the mutated AtR1 3'-UTR was not. The data suggest that miR-155 targets AtR1. Northern blotting detected the hybridization signal of mature miR-155 in HUVECs transfected with miR-155 but not in untransfected cells (Fig. 1). Quantitative real-time PCR (qRT-PCR) and western blotting were used to determine whether miR-155 interfered with AtR1 mRNA expression. According to qRT-PCR, AtR1 mRNA expression was inhib- ited when the miR-155 expression plasmid was transfected into HUVECs $(0.313 \pm 0.050)$, compared with untransfected cells (1.006 \pm 0.076$)$. Western blotting confirmed that AtR1 protein expression was significantly reduced in miR-155-transfected HUVECs $(0.168 \pm 0.045)$ compared with untransfected cells $(0.705 \pm 0.066)$. These data indicate that exogenous miR-155 downregulated the expression of endogenous AtR1 in HUVECs (Fig. 1). 


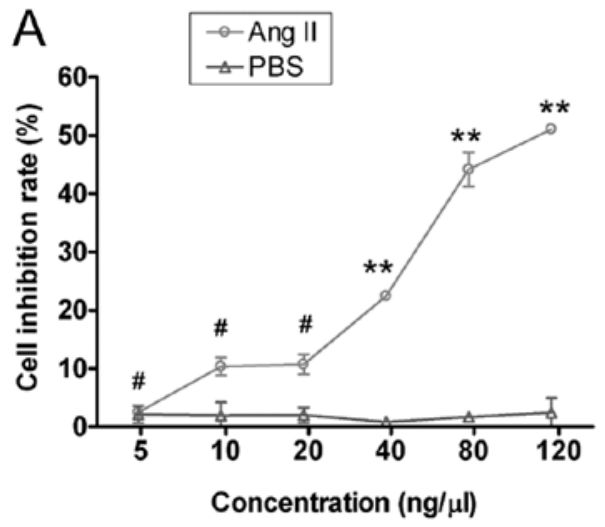

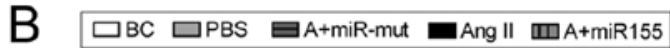

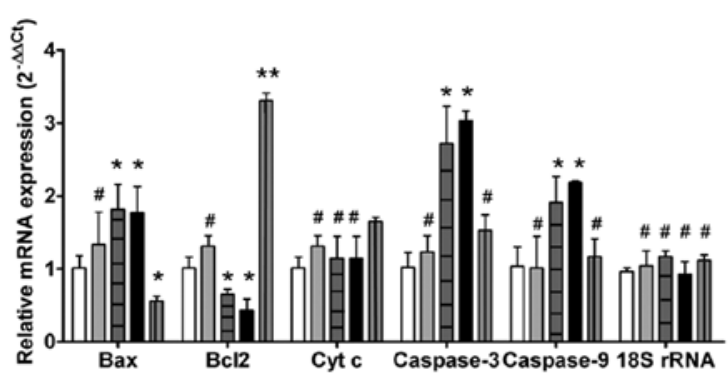

C

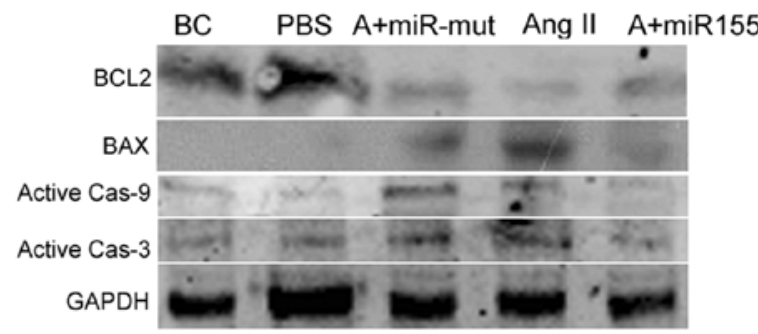

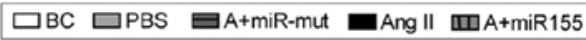

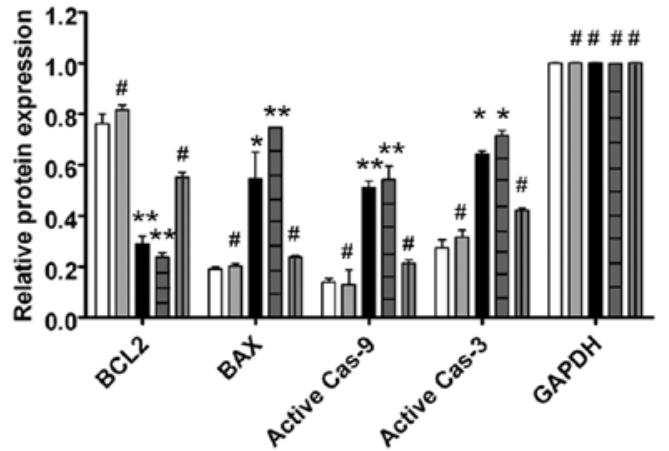

Figure 2. Ang II affects HUVEC proliferation in vitro and miR-155 attenuates that effect. (A) MTT proliferation assay was used to show that exogenous Ang II inhibited HUVEC proliferation in a dose-dependent manner, with a half maximal inhibitory concentration of $68.94 \mathrm{ng} / \mu 1$. ** P $<0.01$ vs. HUVEC-PBS group; ${ }^{\#} \mathrm{P}>0.05$ vs. HUVEC-PBS group; $\mathrm{n}=3$. (B) qRT-PCR showed that mRNA expression of the apoptosis inhibitor Bcl-2 was markedly lower in the HUVEC-Ang II group (Ang II) than in the HUVEC-PBS group (PBS) and the blank control (BC). mRNA expression of the apoptosis-promoting factors Bax, cytochrome $c$, caspases-9 and -3 was markedly higher in the HUVEC-Ang II group (Ang II) than in the PBS and the BC groups. However, after transfection with miR-155, mRNA expression of Bcl-2 in the miR-155-transfected group (A+miR155) was markedly elevated; Bax, cytochrome $c$, caspases-9 and -3 were markedly decreased in this group, but not in the miR-155 mutant-transfected group (A+miR-mut). ${ }^{*} \mathrm{P}<0.05$ vs. $\mathrm{BC} ;{ }^{* *} \mathrm{P}<0.01$ vs. $\mathrm{BC}$; ${ }^{~} \mathrm{P}>0.05$ vs. $\mathrm{BC}$; $\mathrm{n}=3$. (C) Western blotting showed that Bcl-2 protein expression was significantly reduced, but Bax, active caspase- 9 and active caspase-3 were significantly increased in the HUVEC-Ang II group (Ang II) compared with the PBS and the BC groups. Bcl-2 protein expression was significantly increased in the miR-155-transfected group (A+miR155) compared with the HUVEC-Ang II group (Ang II) and the miR-155 mutant-transfected group (A+miR-mut), and Bax, active caspase-9 and active caspase- 3 in this group were markedly decreased. ${ }^{*} \mathrm{P}<0.05$ vs. $\mathrm{BC} ;{ }^{* *} \mathrm{P}<0.01$ vs. $\mathrm{BC} ;{ }^{*} \mathrm{P}>0.05$ vs. $\mathrm{BC} ; \mathrm{n}=3$.

Table II. Detection of mRNA expression of apoptosis-related genes by qRT-PCR.

\begin{tabular}{lccccc}
\hline Gene name & BC & PBS & A+miR-mut & Ang II & A+miR155 \\
\hline Bax & $1.01 \pm 0.16$ & $1.34 \pm 0.44$ & $1.82 \pm 0.34$ & $1.77 \pm 0.36$ & $0.56 \pm 0.07$ \\
Bcl-2 & $1.01 \pm 0.15$ & $1.31 \pm 0.14$ & $0.65 \pm 0.07$ & $0.43 \pm 0.16$ & $3.31 \pm 0.10$ \\
Cyt $c$ & $1.01 \pm 0.15$ & $1.31 \pm 0.14$ & $1.14 \pm 0.30$ & $1.14 \pm 0.30$ & $1.65 \pm 0.05$ \\
Caspase-3 & $1.02 \pm 0.20$ & $1.23 \pm 0.22$ & $2.72 \pm 0.50$ & $3.03 \pm 0.13$ & $1.53 \pm 0.22$ \\
Caspase-9 & $1.03 \pm 0.27$ & $1.01 \pm 0.43$ & $1.91 \pm 0.36$ & $2.19 \pm 0.02$ & $1.17 \pm 0.25$ \\
18S rRNA & $0.96 \pm 0.05$ & $1.04 \pm 0.20$ & $1.17 \pm 0.08$ & $0.92 \pm 0.18$ & $1.12 \pm 0.07$
\end{tabular}

Ang II, angiotensin II; qRT-PCR, quantitative real-time PCR; BC, blank control.

Ang II influences HUVEC proliferation in vitro. To determine whether exogenous Ang II can suppress HUVEC proliferation, inhibition with $0,5,10,20,40,80$ and $120 \mathrm{ng} / \mu \mathrm{l}$ Ang II was measured by the MTT proliferation assay (Fig. 2). There were no significant differences between the HUVEC-Ang II group and the HUVEC-PBS group with Ang II concentrations of $0-20 \mathrm{ng} / \mu \mathrm{l}$ after $24 \mathrm{~h}$. However, with concentrations of 40-120 ng/ $\mu \mathrm{l}$, the HUVEC-PBS group was significantly less susceptible to the proliferation inhibitory effect of Ang II
(Table I). Furthermore, exogenous Ang II suppressed the proliferation of HUVECs in a concentration-dependent manner, with a half maximal inhibitory concentration of $68.94 \mathrm{ng} / \mu \mathrm{l}$ according to the MTT cell proliferation assay.

Exogenous Ang II induces HUVEC damage and apoptosis. To determine whether exogenous Ang II could induce apoptosis in HUVECs, qRT-PCR and western blotting were used to measure the expression levels of apoptosis related 

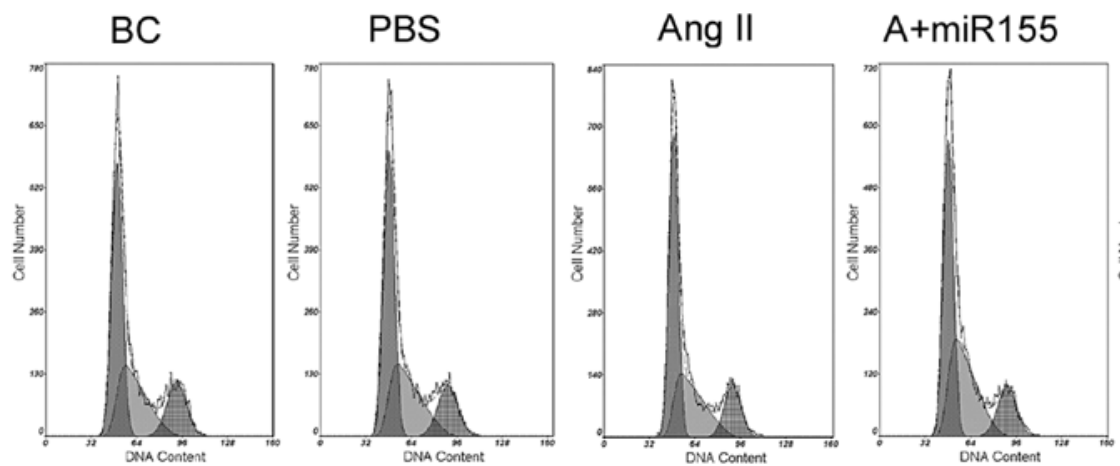

A+miR-mut

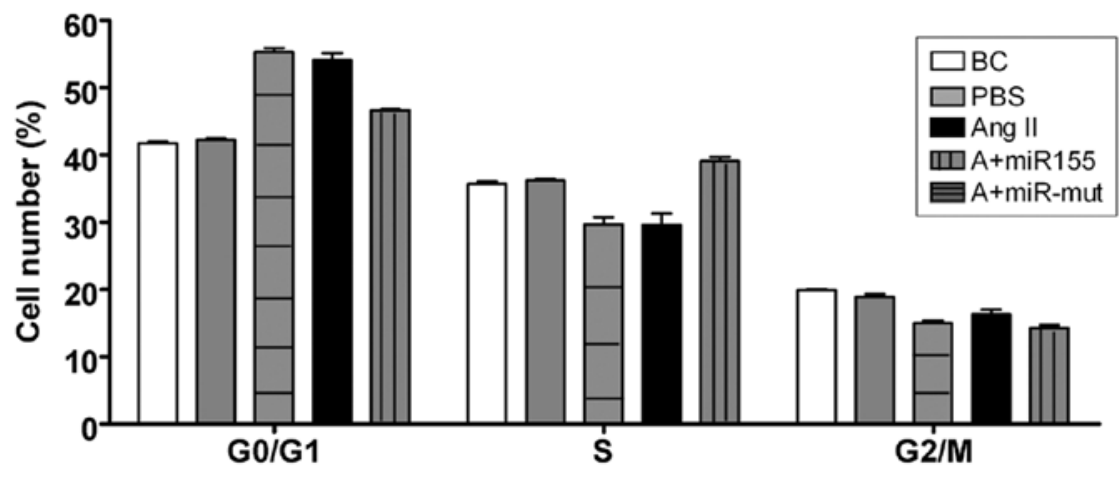

Figure 3. Flow cytometry was used to determine whether Ang II influenced the cell cycle in HUVECs and whether miR-155 could affect this. The HUVEC-Ang II group (Ang II) and the miR-155 mutant-transfected group (A+miR-mut) were arrested in the G0/G1 phase of the cell cycle and the percentage of cells in the $\mathrm{S}$ phase was significantly decreased. By contrast, the cell cycle in the miR-155-transfected group (A+miR155) had more ameliorated.

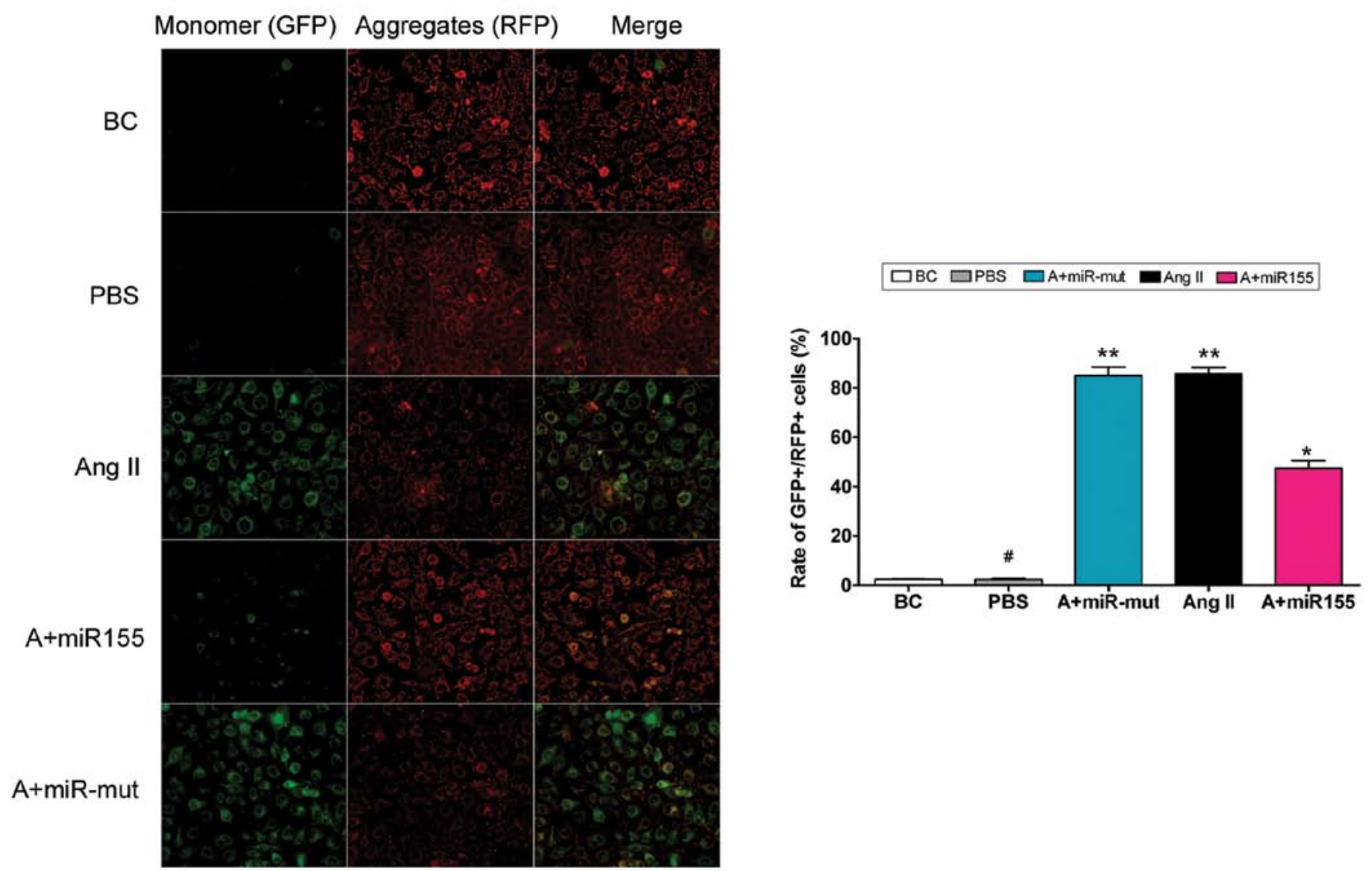

Figure 4. Mitochondrial membrane potential $(\Delta \Psi m)$ of HUVECs was determined by JC-1 staining. $\Delta \Psi m$ changes occurred in the HUVEC-Ang II group (Ang II) and the miR-155 mutant-transfected group (A+miR-mut), suggesting that exogenous Ang II can damage the mitochondria of HUVECs. However, miR-155 attenuated the exogenous Ang II-induced mitochondrial damage in miR-155-transfected HUVECs (A+miR155). ${ }^{*} \mathrm{P}<0.05$ vs. BC group; ${ }^{* *} \mathrm{P}<0.01$ vs. $\mathrm{BC} ;{ }^{*} \mathrm{P}>0.05$ vs. $\mathrm{BC} ; \mathrm{n}=3$. 

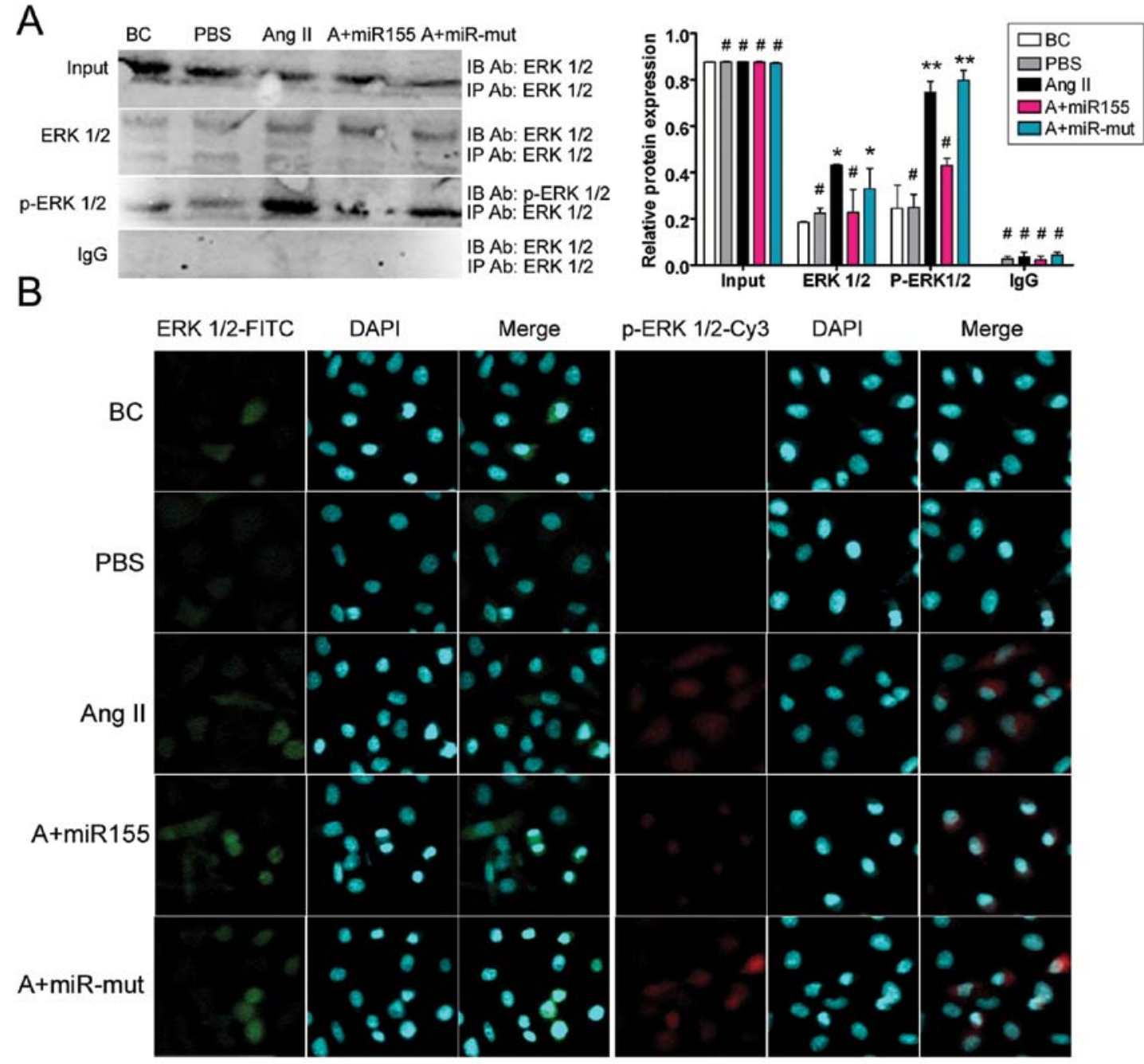

Figure 5. miR-155 reduced the activation and phosphorylation of Ang II-induced ERK1/2. (A) Co-immunoprecipitation western blotting showed no obvious difference in the levels of normal ERK1/2 and phospho-ERK1/2 expression between the blank control (BC) and the HUVEC-PBS group (PBS). However, when exogenous Ang II was added to the culture medium, phospho-ERK1/2 levels were significantly higher in the HUVEC-Ang II group (Ang II) and the miR-155 mutant-transfected group (A+miR-mut) than in the miR-155-transfected group (A+miR155), the BC and the PBS groups. " $\mathrm{P}<0.05$ vs. BC; ${ }^{* * *} \mathrm{P}<0.01 \mathrm{vs}$. BC; ${ }^{\#} \mathrm{P}>0.05$ vs. $\mathrm{BC} ; \mathrm{n}=3$. (B) Immunofluorescence staining showed that ERK1/2 and phospho-ERK1/2 protein expression decreased in miR-155-transfected cells (A+miR155) compared with the HUVEC-Ang II group (Ang II) and miR-155 mutant-transfected cells (A+miR-mut) after exogenous Ang II stimulation.

factors (Fig. 2). qRT-PCR showed that mRNA expression of the apoptosis inhibitor Bcl-2 was markedly lower in the HUVEC-Ang II group than in the HUVEC-PBS group and the blank controls (BCs). By contrast, mRNA expression levels of the apoptosis-promoting factors Bax, cytochrome $c$, caspases-9 and -3 were markedly higher in the HUVEC-Ang II group than in the HUVEC-PBS group and the BCs (Table II). Western blotting confirmed that $\mathrm{Bcl}-2$ protein expression was significantly reduced in the HUVEC-Ang II group compared with the HUVEC-PBS group and the BCs. These data indicate that exogenous Ang II activates the expression of apoptosis related proteins and thereby induces apoptosis.

We used FCM to determine whether Ang II influences the cell cycle in HUVECs. There were no significant changes in the cell cycle distribution of the BCs and the HUVEC-PBS group (Fig. 3). By contrast, the HUVEC-Ang II group was arrested in the G0/G1 phase of the cell cycle and the percentage of cells in the $S$ phase was significantly decreased. These results suggest that exogenous Ang II significantly affects cell cycle regulation in HUVECs in vitro.
To determine whether exogenous Ang II in the cell culture medium damages mitochondria, mitochondrial membrane depolarization was examined. According to previous studies $(15,16)$, normal HUVECs stained with JC-1 emit mitochondrial orange-red fluorescence with a little green fluorescence (Fig. 4). However, compared with the HUVEC-Ang II group, Ang II further decreased the $\Delta \Psi m$ and produced obvious green fluorescence in the HUVEC-PBS group. Thus, exogenous Ang II damages mitochondria in HUVECs.

miR-155 attenuates the effect of exogenous Ang II. To investigate the effect of miR-155 expression, qRT-PCR and western blotting were used to determine the expression levels of apoptosis related factors (Fig. 2). qRT-PCR showed that the mRNA expression of Bcl-2 in miR-155-transfected cells was markedly elevated, whereas Bax, cytochrome $c$, caspases-9 and -3 in this group were markedly decreased (Table II). Western blotting showed that Bcl-2 protein expression was significantly increased in miR-155-transfected cells compared with the HUVEC-Ang II group and the miR-155 mutant-transfected 

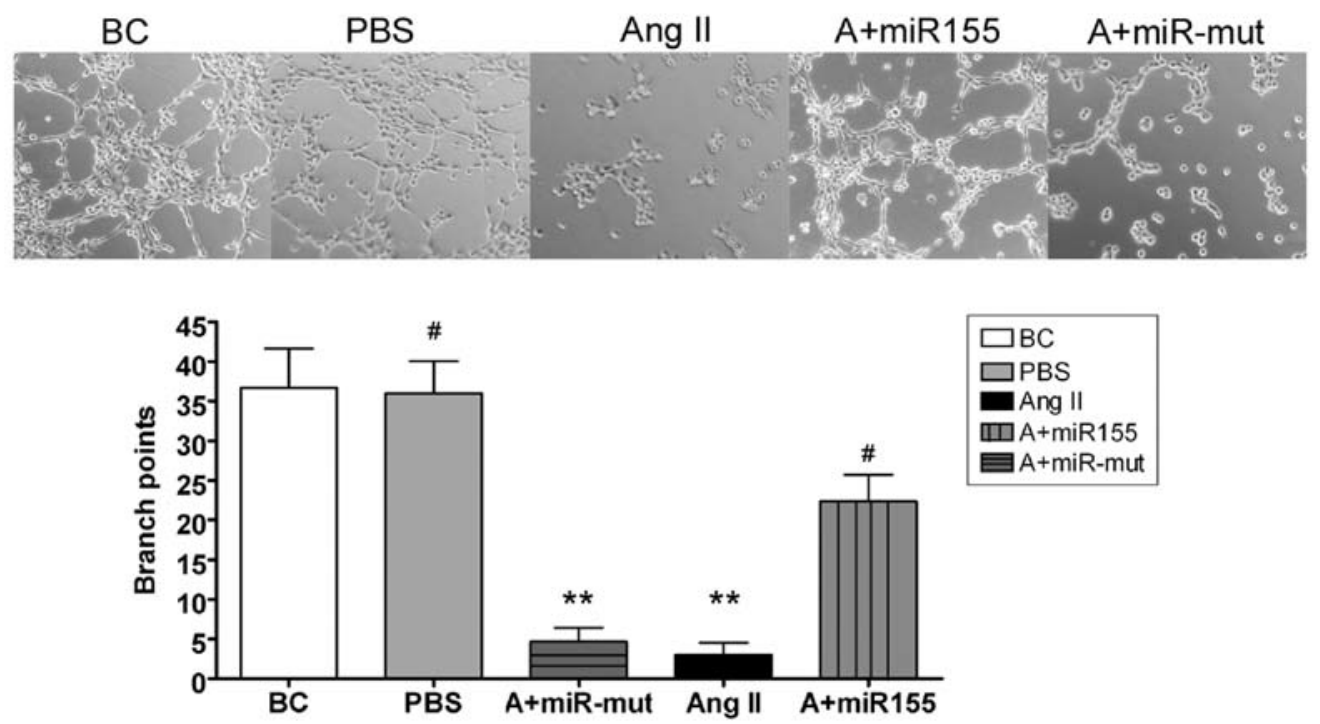

Figure 6. miR-155 maintains the migration and capillary tubule formation abilities of HUVECs. HUVECs formed capillary tubules in migration gels under normal conditions, but migration and capillary tubule formation were markedly reduced when the cell culture medium contained exogenous Ang II. After transfection with miR-155, these abilities were maintained in the presence of Ang II. ${ }^{* *} \mathrm{P}<0.01$ vs. BC; ${ }^{~} \mathrm{P}>0.05$ vs. $\mathrm{BC}$; $\mathrm{n}=6$.

group; Bax, active caspase-9 and active caspase-3 were markedly decreased. These data indicate that miR-155 attenuates the expression of exogenous Ang II-induced apoptosis related proteins. FCM showed no significant differences in cell cycle distribution between the HUVEC-Ang II group and the miR-155 mutant-transfected group (Fig. 3). By contrast, the cell cycle in the miR-155-transfected group was more ameliorated. Compared with the miR-155-transfected group, Ang II further increased $\Delta \Psi m$ and produced obvious orange-red fluorescence but not green fluorescence (Fig. 4). This difference in $\Delta \Psi m$ between the different groups indicates that miR-155 attenuates exogenous Ang II-induced mitochondrial damage in HUVECs.

miR-155 reduces Ang II-induced ERKI/2 activation and phosphorylation. In previous studies, Ang II-induced AtR1 activation was shown to induce ERK1/2 activation and phosphorylation, leading to cellular hypertrophy and apoptosis $(6,7)$. To determine whether miR-155 suppression of At1R expression in HUVECs can reverse the above phenomena, phospho-ERK $1 / 2$ levels were determined by co-immunoprecipitation (co-IP) western blotting. There were no obvious differences in the levels of normal ERK1/2 and phospho-ERK1/2 expression between the BCs and the HUVEC-PBS group (Fig. 5). When exogenous Ang II was added to the culture medium, the levels of phospho-ERK1/2 in the HUVEC-Ang II group and the miR-155 mutant-transfected group were $0.745 \pm 0.048$ and $0.797 \pm 0.043$ of their baseline levels, respectively. These values were significantly higher than those for the miR-155 transfected group, the BCs and the HUVEC-PBS group $(0.430 \pm 0.03,0.244 \pm 0.100$ and $0.249 \pm 0.056$ of baseline, respectively). Immunofluorescence staining showed that ERK1/2 and phospho-ERK1/2 protein expression were both decreased in the miR-155-transfected cells compared with the HUVEC-Ang II group and the miR-155 mutant-transfected cells after stimulation with exogenous Ang II (Fig. 5). The results of co-IP western blotting suggest that miR-155 significantly reduces Ang II-induced activation of the MAPK ERK1/2.
miR-155 maintains HUVEC migration and capillary tubule formation. We also investigated whether exogenous Ang II can influence HUVEC migration and capillary tubule formation in vitro, and whether miR-155 can maintain these abilities. Our experimental results showed that HUVECs can form capillary tubules in migration gels under normal condi-

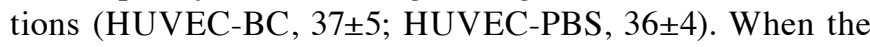
cell culture medium contained exogenous Ang II, HUVEC migration and capillary tubule formation were markedly reduced (HUVEC-Ang II, $3 \pm 2$ ). However, after transfection with miR-155, the migration and capillary tubule formation abilities of the HUVEC were maintained in the presence of Ang II (22 \pm 3 ) (Fig. 6).

\section{Discussion}

Several studies have shown that angiotensin and angiogenic factors in the renin-angiotensin system are crucial markers of cardiovascular function. However, an aberrant or inadequate response to angiotensin-induced stress can cause vascular injury and cardiovascular disease (1-7,15-19). Under normal circumstances, the body regulates excess stress signaling to maintain a dynamic equilibrium. Previous studies have shown changes in the function of miRNAs during stress (3). For example, expression of miR-155 and AtR1 differ between the HUVECs of severely pre-eclamptic pregnant women and those of normal pregnant women (7). In view of this evidence, we investigated whether endogenous miR-155 is specifically expressed to block AtR1, to avoid cell damage from the stress caused by overstimulation with exogenous Ang II. We used HUVECs as a model and simulated them with Ang II. Exogenous Ang II induced HUVEC proliferation in a concentration-dependent manner. One group of HUVECs was then transfected with miR-155. We found that: i) miR-155 maintained cell cycle stability in HUVECs, ensuring normal division and proliferation via suppression of endogenous At1R expression; and, ii) with miR-155 overexpression, HUVEC 
$\Delta \Psi m$ was almost unchanged following stimulation with exogenous Ang II. Thus, miR-155 can maintain mitochondrial stability via suppression of endogenous AtR1 expression to resist Ang II-induced stress. High concentrations of Ang II hindered the ability of HUVECs to form capillary tubules in the extracellular matrix in vitro. However, with overexpression miR-155, HUVEC migration and capillary tubule formation were not influenced by Ang II.

Further experiments showed that exogenous miR-155 can reduce Ang II-induced activation and phosphorylation of ERK1/2 in HUVECs to prevent apoptosis. As is well known, ERK1/2 is an important subfamily of MAPKs that controls a broad range of cellular activities and physiological processes. ERK1/2 is activated transiently or persistently by MEK $1 / 2$ and by upstream MAP3Ks in conjunction with scaffolding proteins and phosphatases, and its activation may have pro-apoptotic functions $(1,19)$. In this study, we found that miR-155 enhances HUVEC growth by inhibiting the MAPK pathway through targeting angiogenic signaling, mainly via AtR1 expression.

In conclusion, HUVECs avoided the damage that may be caused by continuous Ang II-induced stress by selectively targeting miR-155 to silence the AtR1 gene and prevent extracellular Ang II signaling from influencing the intracellular environment. In this manner, cell response to Ang II-induced stress is blocked via suppression of AtR1 by miR-155. miR-155 is thus implicated in pathological angiogenesis in vascular injury and cardiovascular disease, and represents a potential therapeutic target in these disorders.

\section{Acknowledgements}

This study was supported by a grant from the Shanghai Municipal Health Bureau Fund (no. 2010225) and for Traditional Chinese Medicine Scholars (no. 2010L046A) to C.C.

\section{References}

1. Mendell JT and Olson EN: MicroRNAs in stress signaling and human disease. Cell 148: 1172-1187, 2012.

2. Caporali A and Emanueli C: MicroRNA regulation in angiogenesis. Vascul Pharmacol 55: 79-86, 2011.

3. Leung AK and Sharp PA: MicroRNA functions in stress responses. Mol Cell 40: 205-215, 2010.
4. Martin MM, Buckenberger JA, Jiang J, et al: The human angiotensin II type 1 receptor $+1166 \mathrm{~A} / \mathrm{C}$ polymorphism attenuates microRNA-155 binding. J Biol Chem 282: 24262-24269, 2007.

5. Martin MM, Lee EJ, Buckenberger JA, Schmittgen TD and Elton TS: MicroRNA-155 regulates human angiotensin II type 1 receptor expression in fibroblasts. J Biol Chem 281: 18277-18284, 2006.

6. Zheng L, Xu CC, Chen WD, et al: MicroRNA-155 regulates angiotensin II type 1 receptor expression and phenotypic differentiation in vascular adventitial fibroblasts. Biochem Biophys Res Commun 400: 483-488, 2010.

7. Cheng W, Liu T, Jiang F, et al: microRNA-155 regulates angiotensin II type 1 receptor expression in umbilical vein endothelial cells from severely pre-eclamptic pregnant women. Int J Mol Med 27: 393-399, 2011.

8. Cheng W, Liu T, Wan X, Gao Y and Wang H: MicroRNA-199a targets CD44 to suppress the tumorigenicity and multidrug resistance of ovarian cancer-initiating cells. FEBS J 279: 2047-2059, 2012.

9. Liu T, Cheng W, Gao Y, Wang H and Liu Z: Microarray analysis of microRNA expression patterns in the semen of infertile men with semen abnormalities. Mol Med Rep 6: 535-542, 2012.

10. Liu T, Cheng W, Huang Y, Huang Q, Jiang L and Guo L: Human amniotic epithelial cell feeder layers maintain human iPS cell pluripotency via inhibited endogenous microRNA-145 and increased Sox 2 expression. Exp Cell Res 318: 424-434, 2012.

11. Liu T, Chen Q, Huang Y, Huang Q, Jiang L and Guo L: Low microRNA-199a expression in human amniotic epithelial cell feeder layers maintains human-induced pluripotent stem cell pluripotency via increased leukemia inhibitory factor expression. Acta Biochim Biophys Sin (Shanghai) 44: 197-206, 2012.

12. Patrick DM, Montgomery RL, Qi X, et al: Stress-dependent cardiac remodeling occurs in the absence of microRNA-21 in mice. J Clin Invest 120: 3912-3916, 2010.

13. Thum T, Gross C, Fiedler J, et al: MicroRNA-21 contributes to myocardial disease by stimulating MAP kinase signalling in fibroblasts. Nature 456: 980-984, 2008.

14. van Rooij E, Quiat D, Johnson BA, et al: A family of microRNAs encoded by myosin genes governs myosin expression and muscle performance. Dev Cell 17: 662-673, 2009.

15. Zhang XD, Borrow JM, Zhang XY, Nguyen T and Hersey P: Activation of ERK1/2 protects melanoma cells from TRAILinduced apoptosis by inhibiting Smac/DIABLO release from mitochondria. Oncogene 22: 2869-2881, 2003.

16. Wang Z, Tang X, Li Y, et al: 20-Hydroxyeicosatetraenoic acid inhibits the apoptotic responses in pulmonary artery smooth muscle cells. Eur J Pharmacol 588: 9-17, 2008.

17. Markowska AI, Liu FT and Panjwani N: Galectin-3 is an important mediator of VEGF- and bFGF-mediated angiogenic response. J Exp Med 207: 1981-1993, 2010.

18. Henriksen EJ and Prasannarong M: The role of the renin-angiotensin system in the development of insulin resistance in skeletal muscle. Mol Cell Endocrinol: May 4, 2012 (Epub ahead of print).

19. Lu Z and Xu S: ERK1/2 MAP kinases in cell survival and apoptosis. IUBMB Life 58: 621-631, 2006. 